\title{
Note sUR QUELQUES SILPHIDES et LIODIDES de la Collection Grouvelle,
}

par M. G. Portevin.

L'examen d'un magasin de la Collection Grouvelle m'a fourni l'occasion de découvrir quelques Silphides et Liodides nouveaux et de faire les remarques suivantes, qu'il me parait intéressant de faire connaitre.

\section{SILPHID $\nexists$.}

\section{Cholevini.}

Divers Choléviens de la Collection Chevrolat se trouvaient parmi les insectes examinés. Il y avait en particulier des cotypes de Catops spinipes Murray, C. monilis Murray, C. suturalis Murray et C. ascutellaris Murray.

C. spinipes et C. monilis sont des Dissochaetus, comme je l’ai déjà dit (Bull. Mus. Paris, 1902, p. 512). Le premier est une espèce relativement petite (un peu plus de 2 millimètres), d'un brun roussâtre plus foncé sur la tête, le pronotum et la partie postérieure des élytres, la tête restant rousse en avant comme à l'ordinaire. Les tibias sont garnis d'épines grểles, longues et nombreuses, caractère qui rapproche cette espèce de $D$. Geayi Portev. de la Guyane; mais elle en diffère notammeit par la couleur du pronotum et la massue des antennes plus forte.

C. monilis est complètement brun-chàtain, de forme assez allongée, avec le pronotum un peu rétréci à la base, sans être toutefois plus étroit que les élytres, et faiblement impressionné en travers au-devant de la base, comme chez D. curtus Portev. de Bolivie. Il s'en rapproche également par la pubescence plus rase et les éperons terminaux des tibias plus courts qu'à l'ordinaire. Mais il est plus petit, d'une coloration uniforme et d'une forme beaucoup plus allongée.

Un Dissochaetus, qui se trouvait à côté de C. ascutellaris, sous le même nom, me paraît constituer une nouvelle espèce, dont voici la diagnose :

\section{Dissochaetus parallelus nov. sp.}

Oblongo-subparallelus, omnino brunneo-rufescens, basi antennarum, pedibusque dilutioribus. Caput brunneum, antice vix rufescens, minute et dense punctatum, antennis sat crassis, articulo ultimo elongato, sat longe acuminato, 
omnino rufescenti. Pronolum transversum, lateribus antice rotundatis, postice vix angustior, angulis posticis subobtusis, ad basin transversaliter haud impressionatum. Elytra ad basin subparallela, deinde sat longe rotundatim attenuata, leviter et dense transversim strigosa. Pedes rubro testacei, tibiis parum et longe spinosis. Long. 2 millim. 3.

Voisin de monilis, mais sensiblement plus petit, avec un pronotum non impressionıé en travers à la base, et les strigosités des élytres fines, serrées, peu visibles.

1 seul exemplaire $\sigma^{*}$ Venezuela : Caracas (Sallé).

Catops suturalis Murray = Nemadus suturalis. Derbent (Motschoulsky).

Catops ascutellaris Murray $=$ Ptomaphagus ascutellaris. Venezuela : Ciaracas (Sallé).

Les Choléviens de la Collection Grouvelle comprenaient deux espèces nouvelles :

\section{Dissochaetus calcaratus nov. sp.}

Ovatus, omnino rufescens, capite brunneo, antennis in medio infuscatis, pedibus rufo-testaceis. Caput minute et dense punctatum, antice vix dilutior, antennis sat crassis, articulis $6^{\circ}-10^{\circ}$ infuscatis, ultimo luteo, elongato et acuminato. Pronotum fortiter transversum, vix postice, antice paulo angustatum, omnibus angulis rectis ad apicem rotundatis. Elytra transversim leviter et dense strigosa. Pedes rufo-testacei, tibiis parum et curte spinosis, longissime ad apicem calcaratis. Long. 2 millim. 5 à 2 millim. 9 .

Celte espèce, qui est longuement pubescente de gris, doit être proche de D. exigous Kirsch et D. oval is Kirsch, du Pérou. Elle en diffère, outre sa coloration, par les éperons des tibias, notamment aux postérieurs, où le plus long dépasse visiblement le premier article des tarses.

Brésil : Bahia (Fruhstorfer).

\section{Ptomaphagus angusticornis nov. sp.}

Brunneo-rufescens, capite antice rufo, elytris ad apicem dilutioribus, antennarum basi rubra, articulo ultimo ad apicem luteo. Oblongus, parum convexus, sat nitidus, antennis rufo-brunneis, clava infumata angustissima, articulo ultimo sat longe acuminato. Pronotum lateribus antice regulariter rotundatis, angulis posticis acutis el prolongatis. Elytra transversim, leviter, sat dense, parum oblique strigosa, ad apicem parum truncata, stria suturali integra. Pedes rubro-brunnei, tarsis posterioribus elongatis, tibiis equalibus, articulo primo $2^{\circ}-3^{\circ}$ que simul aquali. 2 millim. 5 .

1 \& Java oriental = Monts Tengger. 4,00o mètres (Fruhstorfer). 
Évidemment voisin de $P$. cilipes Port. de l'Inde méridionale. Il s'en distingue par la couleur et la forme de ses antennes, qui sont entièrement rousses chez cilipes, avec une massue épaisse dont le dernier article est brièvement et obtusément acuminé; ce dernier a en outre le pronotum avec des angles postérieurs presque droits, à peine prolongés, et les élytres très obliquement striolés. Nul doute que l'examen du ơ ne fournisse des caractères distinctifs encore plus tranchés.

C'est le premier Cholévien connu des Îles de la Sonde. On remarquera qu'il provient, comme d'ailleurs les Liodides de la même contrée décrits plus loin, d'une altitude élevée.

\section{SILPHINI.}

J'ai décrit (Bull. du Mus., 1920, p. 505) sous le nom d'Eusilpha (Calosilpha) Renatae, un Silphide des l̀les Malaises. Cette indication assez vague se trouve précisée par la provenance de deux nouveaux exemplaires; l'espèce appartient aux Ìles Célèbes.

Je profite de la circonstance pour établir une nouvelle classification de ce groupe de Silphini métalliques. Les sous-genres que javais établis (Bull. Mus., 1920, p. 396) doivent être élevés au rang de genres, et une nouvelle coupe générique doit être admise pour le groupe de viridis Mots. Voici d'ailleurs un tableau dichotomique de ces genres :

1. Pronotum profondément échancré en demi-hexagone en avant. Corps unicolore, épais, d'un noir bleuâtre en dessus, généralement plus franchement bleu en dessous, surtout sur les épipleures. Élytres présentant dans l'intervalle externe un renflement longitudinal simulant une quatrième côte, moins accentué et quelquefois absent chez la . Extrémité plus ou moins tronquée arrondie $\sigma^{\Upsilon}$, prolongée sinuée ․

Eusilpha Sem.

1'. Pronotum échancré en avant en courbe plus ou moins plate. Corps déprimé. Intervalle externe des élytres sans trace de renflement. Pronotum plus ou moins rouge orangé.

2. Elytres ne présentant pratiquement que denx côtes, l'externe à peu près totalement oblitérée. Élytres atténués en arrière et échancrés

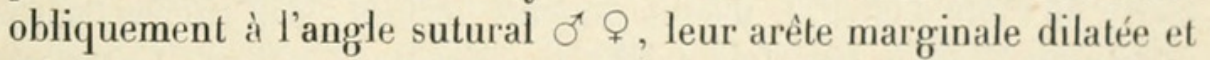
relevée sur toute sa longueur en lame verticale. Fémurs antérieurs et intermédiaires ơ échancrés dentés en dessous à l'extrémité.

Deutosilpha Port.

2'. Élytres avec 3 côtes bien visibles. $\sigma^{\top}$ Tous les fémur's simples.

3. Élytres à arête marginale simple dans les deux sexes, à gouttière latérale large, leur extrémité nettement tronquée bisinuée. Calosilpha Port. 
3'. Arête marginale simple chez la 우, dilatée en lame relevée à partir du milieu en arrière $\sigma^{\top}$. Élytres atténués, tronqués obliquement ou plus ou moins sinués, à goultière marginale étroite.

Chrysosilpha gen. nov.

Il est à remarquer que les espèces de ce groupe, à très peu d'exceptions près, ont, à l'angle suluro-apical, une petite pointe aiguë. Les deux seules espèces faisant exception à cette règle sont C. formosa Cast. et C. chloroptera Cast. qui ont été réunis à tort par les auteurs.

Comme complément à la description de $C$. Renatae, voici quelques détails sur cette espèce.

Le pronotum est fortement impressionné sur la ligne médiane, laquelle n'est pas marquée chez les deux espèces voisines C.viridis Mots. et C. calestis Dohrn. Cette impression est fortement poncluée de même que les côtés et la base. Il est également moins transverse, moins arrondi sur les côtés et avec les angles postérieurs mieux marqués. Les élytres ont les deux còtes internes très convergentes en arrière, à peu près comme chez viridis. Chez calestis, elles le sont très peu; de plus, la rôte interne est très peu courbée vers l'extérieur en avant, tandis qu'elle l'est très sensiblement chez viridis et encore plus chez Renatae. L'extrémité des élytres du $\sigma^{x}$, chez celte dernière espèce, est émarginée obliquement vers la suture, cette troncature étant très légèrement concave. de sorte que les deux extrémités forment des angles vifs.

Les $f$ de celestis et de viridis ont les élytres atténués, légèrement prolongés sinués à l'apex, puis émarginés vers la sulure, de sorte que leur extrémité offre un lobe anguleux émoussé à la pointe.

J'ai dit plus haut que $C$. Jormosa et $C$. chloroptera avaient été réunis à tort. En effet, C. chloroptera a le pronotum moins transverse, moins fortement rétréci en avant, les taches plus grandes, les antérieures n'étant éloignnées que du diamètre de l'une d'elles, le disque beaucoup moins ponctué. Les élytres, dont la ponctuation est plus fine. sont d'un vert bleuâtre, avec une tendance à tourner au vert; l'insecte est parfois d'un vert doré éclatant, en dessus et en dessous (var. magnifica nov.).

C. formosa a le pronotum plus transverse, plus fortement rétréci en avant, les taches plus petites, les antérieures éloignées du double du diamètre de l'une d'elles, le disque ponctué partout, sauf sur les tachıs, cette ponctuation étant toutefois faible et écartée au milieu. Les élytres ont la ponctuation plus forte et sont d'un bleu plus franc. 


\section{$2 \mathrm{BHL}$ Biodiversity Heritage Library}

1921. "Note sur quelques Silphides et Liodides de la Collection Grouvelle." Bulletin du

Muse

um national d'histoire naturelle 1921, 535-538.

https://doi.org/10.5962/bhl.part.27742.

View This Item Online: https://www.biodiversitylibrary.org/item/27554

DOI: https://doi.org/10.5962/bhl.part.27742

Permalink: https://www.biodiversitylibrary.org/partpdf/27742

\section{Holding Institution}

New York Botanical Garden, LuEsther T. Mertz Library

\section{Sponsored by}

MSN

\section{Copyright \& Reuse}

Copyright Status: NOT_IN_COPYRIGHT

This document was created from content at the Biodiversity Heritage Library, the world's largest open access digital library for biodiversity literature and archives. Visit BHL at https://www.biodiversitylibrary.org. 